\title{
Possibility Fuzzy Soft Expert Set
}

Maruah Bashir

School of Mathematical Sciences, Faculty of Science and Technology, Universiti Kebangsaan Malaysia, 43600 UKM Bangi, Selangor DE, Malaysia aabosefe@yahoo.com

\author{
Abdul Razak Salleh \\ School of Mathematical Sciences, Faculty of Science and \\ Technology, Universiti Kebangsaan Malaysia, 43600 UKM \\ Bangi, Selangor DE, Malaysia \\ aras@ukm.my
}

\begin{abstract}
In this paper we introduce the concept of possibility fuzzy soft expert set .We also define its basic operations, namely complement, union, intersection, AND and OR, and study some of their properties. Finally, we give an application of this theory in solving a decision making problem.
\end{abstract}

Keywords - Fuzzy soft expert set, possibility fuzzy soft set, possibility fuzzy soft expert set

\section{Introduction}

In 1999 Molodtsov [1] initiated a novel concept of soft set theory as a new mathematical tool for dealing with uncertainties . After Molodtsov's work, some different operations and application of soft sets were studied by Chen et al. [2] and Maji et al. [3,4]. Furthermore Maji et al. [5] presented the definition of fuzzy soft set as a generalization of Molodtsov's soft set. Roy and Maji [6] gave an application of this concept in decision making problem. In 2010 Çağman et al. introduced the concept of fuzzy parameterized fuzzy soft sets and their operations [7]. Alkhazaleh et al. [8] generalized the concept of fuzzy soft set to possibility fuzzy soft set and they gave some applications of this concept in decision making and medical diagnosis. They also introduced the concept of fuzzy parameterized interval-valued fuzzy soft set [9], where the mapping in which the approximate functions are defined from fuzzy parameters set to the interval-valued fuzzy subsets of universal set, and gave an application of this concept in decision making. Alkhazaleh and Salleh [10] introduced the concept of soft expert sets where the user can know the opinion of all experts in one model and gave an application of this concept in decision making problem. Salleh et al. [11] introduced the concept of multi paramatriezed soft set and studied its properties and basic operations. Alkhazaleh and Salleh [12] introduced the concept of fuzzy soft expert sets and gave an application of this concept in a decision making problem. In this paper we introduce the concept of possibility fuzzy soft expert set which is a combination of possibility fuzzy soft set and fuzzy soft expert set. We also define its basic operations namely complement, union, intersection, AND and OR. Finally,we give an application of this concept in adecision making problem.

\section{Preliminaries}

In this section we recall some definitions and properties regarding soft expert set, fuzzy soft expert set and possibility fuzzy soft set.
Alkhazaleh and Salleh [10] defined soft expert set and in [12] Alkhazaleh and Salleh defined a fuzzy soft expert set in the following way. Let $U$ be a universe, $\mathrm{E}$ a set of parameters, and $X$ a set of experts (agents). Let $O$ be a set of opinions, $Z=E \times X \times O$ and $A \subseteq Z$.

Definition 2.1. [12] A pair $(F, A)$ is called a soft expert set over $U$, where $F$ is a mapping $F: A \rightarrow P(U)$ and $P(U)$ denotes the power set of $U$.

Definition 2.2. [12] A pair $(F, A)$ is called a fuzzy soft expert set over $U$, where $F$ is a mapping $F: A \rightarrow I^{U}$, and $I^{U}$ denotes all fuzzy subsets of $U$.

Definition 2.3. [12] The complement of a fuzzy soft expert set $(F, A)$ is denoted by $(F, A)^{c}$ and is defined by $(F, A)^{c}=\left(F^{c},{ }^{\prime} \mathrm{I} A\right)$ where $F^{c}:{ }^{\prime} \mathrm{I} A \rightarrow p(U)$ is a mapping given by $F^{c}(\alpha)=c(F($ 'I $\alpha)), \forall \alpha \in$ 'I $A$, where $c$ is a fuzzy complement.

Definition 2.4. [12] The union of two fuzzy soft expert sets $(F, A)$ and $(G, B)$ over $U$, denoted by $(F, A) \tilde{U}(G, B)$, is a fuzzy soft expert set $(H, C)$ where $C=A \cup B$ and $\forall \varepsilon \in C$,

$$
H(\varepsilon)= \begin{cases}F(\varepsilon) & \text { if } \varepsilon \in A-B, \\ G(\varepsilon) & \text { if } \varepsilon \in B-A, \\ s(F(\varepsilon), G(\varepsilon)) & \text { if } \varepsilon \in A \cap B,\end{cases}
$$

where $s$ is an $s$-norm.

Definition 2.5. [12] The intersection of two fuzzy soft expert sets $(F, A)$ and $(G, B)$ over $U$, denoted by $(F, A) \tilde{\cap}(G, B)$, is a soft expert set $(H, C)$ where $C=A \cup B$ and $\forall \varepsilon \in C$, 


$$
H(\varepsilon)= \begin{cases}F(\varepsilon) & \text { if } \varepsilon \in A-B, \\ G(\varepsilon) & \text { if } \varepsilon \in B-A, \\ t(F(\varepsilon), G(\varepsilon)) & \text { if } \varepsilon \in A \cap B,\end{cases}
$$

where $t$ is a $t$-norm.

The following definitions are due to Alkhazaleh et al. [8].

Definition 2.6. Let $U=\left\{x_{1}, x_{2}, \ldots, x_{n}\right\}$ be the universal set of elements and $E=\left\{e_{1}, e_{2}, \ldots, e_{m}\right\}$ be the universal set of parameters. The pair $(U, E)$ will be called a soft universe. Let $F: E \rightarrow I^{U}$ and $\mu$ be a fuzzy subset of $E$, i.e. $\mu: E \rightarrow I^{U}$, where $I^{U}$ is the collection of all fuzzy subsets of $U$. Let $F_{\mu}: E \rightarrow I^{U} \times I^{U}$ be a function defined as follows:

$$
F_{\mu}(e)=(F(e)(x), \mu(e)(x)), \forall x \in U .
$$

Then $F_{\mu}$ is called a possibility fuzzy soft set (PFSS in short) over the soft universe $(U, E)$. For each parameter $e_{i}, F_{\mu}\left(e_{i}\right)=\left(F\left(e_{i}\right)(x), \mu\left(e_{i}\right)(x)\right)$ indicates not only the degree of belongingness of the elements of $U$ in $F\left(e_{i}\right)$, but also the degree of possibility of belongingness of the elements of $U$ in $F\left(e_{i}\right)$, which is represented by $\mu\left(e_{i}\right)$. So we can write $F_{\mu}\left(e_{i}\right)$ as follows:

$$
F_{\mu}\left(e_{i}\right)=\left\{\left(\frac{x_{1}}{F\left(e_{i}\right)\left(x_{1}\right)}, \mu\left(e_{i}\right)\left(x_{1}\right)\right), \ldots,\left(\frac{x_{n}}{F\left(e_{i}\right)\left(x_{n}\right)}, \mu\left(e_{i}\right)\left(x_{n}\right)\right)\right\} \text {. }
$$

Sometime we write $F_{\mu}$ as $\left(F_{\mu}, E\right)$. If $A \subseteq E$ we can also have a PFSS $\left(F_{\mu}, A\right)$.

Definition 2.7. Union of two PFSSs $F_{\mu}$ and $G_{\delta}$, denoted by $F_{\mu} \tilde{U} G_{\delta}$, is a PFSS $H_{v}: E \rightarrow I^{U} \times I^{U}$ defined by

$$
H_{v}(e)=(H(e)(x), v(e)(x)), \forall e \in E
$$

such that $H(e)=s(F(e), G(e))$ and $v(e)=s(\mu(e), \delta(e))$ where $s$ is an $s$-norm.

Definition 2.8. Intersection of two PFSSs $F_{\mu}$ and $G_{\delta}$, denoted by $F_{\mu} \tilde{\cap} G_{\delta}$, is a PFSS $H_{v}: E \rightarrow I^{U} \times I^{U}$ defined by

$$
H_{v}(e)=(H(e)(x), v(e)(x)), \forall e \in E
$$

such that $H(e)=t(F(e), G(e)) \quad$ and $v(e)=t(\mu(e), \delta(e))$ where $t$ is a $t$-norm.

\section{Possibility Fuzzy Soft Expert Sets}

In this section we generalise the concept of fuzzy soft expert sets as introduced by Alkhazaleh and Salleh. [12]. In our generalisation of fuzzy soft expert set, a possibility of each element in the universe is attached with the parameterization of fuzzy sets while defining a fuzzy soft expert set.

Definition 3.1. Let $U=\left\{u_{1}, u_{2}, \ldots, u_{n}\right\}$ be the universal set of elements, $E=\left\{e_{1}, e_{2}, \ldots, e_{m}\right\}$ be the universal set of parameters, $X$ be a set of experts, and $O=\{1=$ agree, $0=$ disagree $\}$ a set of opinions. Let $Z=\{E \times X \times O\}$ and $A \subseteq Z$. Let $F: Z \rightarrow I^{U}$ and $\mu$ be a fuzzy subset of $Z$, i.e. $\mu: Z \rightarrow I^{U}$, where $I^{U}$ is the collection of all fuzzy subsets of $U$. Let $F_{\mu}: Z \rightarrow I^{U} \times I^{U}$ be a function defined as follows:

$$
F_{\mu}(z)=(F(z)(u), \mu(z)(u)), \forall u \in U .
$$

Then $F_{\mu}$ is called a possibility fuzzy soft expert set (PFSES in short) over the soft universe $(U, Z)$. For each $z_{i}, F_{\mu}\left(z_{i}\right)=\left(F\left(z_{i}\right)(u), \mu\left(z_{i}\right)(u)\right)$ indicates the degree of belongingness of the elements of $U$ in $F\left(z_{i}\right)$, and also the degree of possibility of such belongingness which is represented by $\mu\left(z_{i}\right)$. So we can write $F_{\mu}\left(z_{i}\right)$ as follows:

$$
F_{\mu}\left(z_{i}\right)=\left\{\left(\frac{u_{1}}{F\left(z_{i}\right)\left(u_{1}\right)}, \mu\left(z_{i}\right)\left(u_{1}\right)\right), \ldots,\left(\frac{u_{n}}{F\left(z_{i}\right)\left(u_{n}\right)}, \mu\left(z_{i}\right)\left(u_{n}\right)\right)\right\} .
$$

Sometime we write $\left(F_{\mu}, Z\right)$ as $F_{\mu}$. If $A \subseteq Z$ we can also have a PFSES $\left(F_{\mu}, A\right)$.

Definition 3.2. Let $\left(F_{\mu}, A\right)$ and $\left(G_{\delta}, B\right)$ be two PFSESs over $(U, Z) \cdot\left(F_{\mu}, A\right)$ is said to be a possibility fuzzy soft expert subset (PFSE subset) of $\left(G_{\delta}, B\right)$, and we write $\left(F_{\mu}, A\right) \subseteq\left(G_{\delta}, B\right)$, if $A \subseteq B$ and $\forall \varepsilon \in A$,

i. $\mu(\varepsilon)$ is a fuzzy subset of $\delta(\varepsilon)$,

ii. $F(\varepsilon)$ is a fuzzy subset of $G(\varepsilon)$.

Definition 3.3. A possibility agree - fuzzy soft expert $\operatorname{set}\left(F_{\mu}, A\right)$ over $U$ is a possibility fuzzy soft expert subset of $\left(F_{\mu}, A\right)$ defined as follows :

$$
\left(F_{\mu}, A\right)_{1}=(F(\alpha), \mu(\alpha)) \text {, where } \alpha \in E \times X \times\{1\} .
$$

Definition 3.4. A possibility disagree - fuzzy soft expert $\operatorname{set}\left(F_{\mu}, A\right)_{0}$ over $U$ is a possibility fuzzy soft expert subset of $\left(F_{\mu}, A\right)$ defined as follows :

$$
\left(F_{\mu}, A\right)_{0}=(F(\alpha), \mu(\alpha)) \text {, where } \alpha \in E \times X \times\{0\} .
$$


Definition 3.5. Let $\left(F_{\mu}, A\right)$ be a PFSES over $(U, Z)$. Then the complement of $\left(F_{\mu}, A\right)$, denoted by $\left(F_{\mu}, A\right)^{c}$ is defined by $\left.\left(F_{\mu}, A\right)^{c}=(\tilde{c}(F(\eta \alpha)), c(\mu(\eta \alpha))) \forall \alpha \in\right\rceil^{A}$, where $\tilde{c}$ is a fuzzy soft expert complement and $c$ is a fuzzy complement.

Definition 3.6. Union of two PFSESs $\left(F_{\mu}, A\right)$ and $\left(G_{\delta}, B\right)$ over $U$, denoted by $\left(F_{\mu}, A\right) \tilde{U}\left(G_{\delta}, B\right)$, is a PFSES $\left(H_{v}, C\right)$ where $C=A \cup B$, is defined by

$$
v(\alpha)=s(\mu(\alpha), \delta(\alpha)), \forall \alpha \in C
$$

and

$$
H(\alpha)=F(\alpha) \tilde{U} G(\alpha), \forall \alpha \in C
$$

where $s$ is an $s$-norm and $\tilde{U}$ is a fuzzy soft expert union.

Definition 3.7. Intersection of two PFSESs $\left(F_{\mu}, A\right)$ and $\left(G_{\delta}, B\right)$ over $U$, denoted by $\left(F_{\mu}, A\right) \tilde{\cap}\left(G_{\delta}, B\right)$, is a PFSES $\left(H_{v}, C\right)$ where $C=A \cup B$, is defined by

$$
v(e)=t(\mu(e), \delta(e)), \forall e \in C
$$

and

$$
H(e)=F(e) \tilde{\cap} G(e), \forall e \in C
$$

where $t$ is a $t$-norm and $\tilde{\cap}$ is a fuzzy soft expert intersection.

\section{An application of possibility fuzzy soft expert set}

Ahkhazaleh and Salleh [12] applied the theory of fuzzy soft expert sets to solve a decision making problem. In this section, we present an application of PFSES in a decision making problem by generalizing Ahkhazaleh and Salleh's algorithm to be compatible with our work.

The problem we consider is as below.

Suppose that electricity board wants to make an electric generator using waterfalls. Suppose there are three different locations and they want to take the opinion of some experts concerning these locations. Let $U=\left\{u_{1}, u_{2}, u_{3}\right\}$ be a set of locations, $E=\left\{e_{1}, e_{2}, e_{3}, e_{4}\right\}$ a set of decision parameters where $e_{i}(i=1,2,3,4)$ denotes the decision "Ebb and tide," "wind power," "the power of the regression" and "solar energy" respectively, and let $X=(q, m)$ be a set of experts (two members). Suppose that the electricity board wants to choose one such location depending on the parameters. After a serious discussion the committee constructs the following possibility fuzzy soft expert set:

$$
\begin{aligned}
\left(F_{\mu}, Z\right)=\{ & \left\{\left(e_{1}, q, 1\right),\left\{\left(\frac{u_{1}}{0.4}, 0.3\right),\left(\frac{u_{2}}{0.2}, 0.2\right),\left(\frac{u_{3}}{0.5}, 0.3\right)\right\}\right\}, \\
& \left\{\left(e_{2}, q, 1\right),\left\{\left(\frac{u_{1}}{0.1}, 0.3\right),\left(\frac{u_{2}}{0.2}, 0.4\right),\left(\frac{u_{3}}{0.7}, 0.3\right)\right\}\right\}, \\
& \left\{\left(e_{4}, q, 1\right),\left\{\left(\frac{u_{1}}{0.9}, 0.1\right),\left(\frac{u_{2}}{0.6}, 0.4\right),\left(\frac{u_{3}}{0.1}, 0.5\right)\right\}\right\}, \\
& \left\{\left(e_{1}, m, 1\right),\left\{\left(\frac{u_{1}}{0.8}, 0.1\right),\left(\frac{u_{2}}{0.3}, 0\right),\left(\frac{u_{3}}{0.3}, 0.2\right)\right\}\right\}, \\
& \left\{\left(e_{2}, m, 1\right),\left\{\left(\frac{u_{1}}{0.1}, 0.1\right),\left(\frac{u_{2}}{0}, 0.5\right),\left(\frac{u_{3}}{0.2}, 0.1\right)\right\}\right\}, \\
& \left\{\left(e_{3}, m, 1\right),\left\{\left(\frac{u_{1}}{0.1}, 0\right),\left(\frac{u_{2}}{0.3}, 0\right),\left(\frac{u_{3}}{0.1}, 0.1\right)\right\}\right\}, \\
& \left\{\left(e_{1}, q, 0\right),\left\{\left(\frac{u_{1}}{0.1}, 0.2\right),\left(\frac{u_{2}}{0.6}, 0.3\right),\left(\frac{u_{3}}{0.2}, 0.1\right)\right\}\right\}, \\
& \left\{\left(e_{2}, q, 0\right),\left\{\left(\frac{u_{1}}{0.3}, 0.5\right),\left(\frac{u_{2}}{0.6}, 0.5\right),\left(\frac{u_{3}}{0.5}, 0.1\right)\right\}\right\}, \\
& \left\{\left(e_{3}, q, 0\right),\left\{\left(\frac{u_{1}}{0.2}, 0.2\right),\left(\frac{u_{2}}{0.4}, 0.3\right),\left(\frac{u_{3}}{0.5}, 0.1\right)\right\}\right\}, \\
& \left\{\left(e_{1}, m, 0\right),\left\{\left(\frac{u_{1}}{0.3}, 0.3\right),\left(\frac{u_{2}}{0.5}, 0.5\right),\left(\frac{u_{3}}{0.4}, 0.3\right)\right\}\right\}, \\
& \left\{\left(e_{3}, m, 0\right),\left\{\left(\frac{u_{1}}{0.2}, 0.3\right),\left(\frac{u_{2}}{0.4}, 0.7\right),\left(\frac{u_{3}}{0.1}, 0.3\right)\right\}\right\}, \\
& \left.\left\{\left(e_{4}, m, 0\right),\left\{\left(\frac{u_{1}}{0.2}, 0.1\right),\left(\frac{u_{2}}{0.3}, 0.1\right),\left(\frac{u_{3}}{0.4}, 0.1\right)\right\}\right\}\right\},
\end{aligned}
$$

The following algorithm may be followed by the electricity board to determine the best location.

1. Input the PFSES $(F, Z)$.

2. Find the highest numerical grade for the agree-PFSES and disagree-PFSES.

3. Compute the score of each such locations by taking the sum of the products of these numerical grades with the corresponding possibility $\lambda$, for the agree-PFSES which is denoted by $\left(A_{j}\right)$ and disagree-PFSES denoted by $\left(D_{j}\right)$.

4. Find $s_{j}=A_{j}-D_{j}$.

5. Find $m$, for which $s_{m}=\max s_{j}$. Then $s_{m}$ is the highest score. If $m$ has more than one value, then any one of them could be chosen by the electricity board using its option.

Now we use this algorithm to find the best choice for the electricity board to make an electric generator. 
TABLE I. GRADE FOR AGREE PFSES

\begin{tabular}{c|ccc}
\hline$H$ & $u_{i}$ & Highest numerical grade & $\lambda_{i}$ \\
\hline$\left(e_{1}, q\right)$ & $u_{3}$ & 0.5 & 0.3 \\
$\left(e_{2}, q\right)$ & $u_{3}$ & 0.7 & 0.3 \\
$\left(e_{4}, q\right)$ & $u_{1}$ & 0.9 & 0.1 \\
$\left(e_{1}, m\right)$ & $u_{1}$ & 0.8 & 0.1 \\
$\left(e_{2}, m\right)$ & $u_{3}$ & 0.2 & 0.1 \\
$\left(e_{3}, m\right)$ & $u_{2}$ & 0.3 & 0 \\
\hline
\end{tabular}

score $\left(u_{1}\right)=(0.9 \times 0.1)+(0.8 \times 0.1)=0.17$

score $\left(u_{2}\right)=(0.3 \times 0)=0$

score $\left(u_{3}\right)=(0.5 \times 0.3)+(0.7 \times 0.3)+(0.2 \times 0.1)=0.38$

TABLE II. GRADE FOR DIS-AGREE PFSES

\begin{tabular}{c|ccc}
\hline$H$ & $u_{i}$ & Highest numerical grade & $\lambda_{i}$ \\
\hline$\left(e_{1}, q\right)$ & $u_{2}$ & 0.6 & 0.3 \\
$\left(e_{2}, q\right)$ & $u_{2}$ & 0.6 & 0.5 \\
$\left(e_{3}, q\right)$ & $u_{3}$ & 0.5 & 0.1 \\
$\left(e_{1}, m\right)$ & $u_{2}$ & 0.5 & 0.5 \\
$\left(e_{3}, m\right)$ & $u_{2}$ & 0.4 & 0.7 \\
$\left(e_{4}, m\right)$ & $u_{3}$ & 0.4 & 0.1 \\
\hline
\end{tabular}

score $\left(u_{1}\right)=0$

score $\left(u_{2}\right)=(0.6 \times 0.3)+(0.6 \times 0.5)+(0.5 \times 0.5)+(0.4 \times 0.7)=1.01$

score $\left(u_{3}\right)=(0.5 \times 0.1)+(0.4 \times 0.1)=0.09$

For abbreviation suppose that

$A_{j}=$ the score of each numerical grade for agree-PFSES,

$D_{j}=$ the score of each numerical grade for dis-agree-PFSES.

TABLE III. $\quad s_{j}=A_{j}-D_{j}$

\begin{tabular}{ccc}
\hline$A_{j}$ & $D_{j}$ & $s_{j}=A_{j}-D_{j}$ \\
\hline score $\left(u_{1}\right)=0.17$ & score $\left(u_{1}\right)=0$ & 0.17 \\
score $\left(u_{2}\right)=0$ & score $\left(u_{2}\right)=1.01$ & -1.01 \\
$\operatorname{score}\left(u_{3}\right)=0.38$ & score $\left(u_{3}\right)=0.09$ & 0.29 \\
\hline
\end{tabular}

Then $\max s_{j}=s_{3}$, so the electricity board will select the location with the highest score. Hence, they will choose location $u_{3}$.

\section{Acknowledgement}

The authors would like to acknowledge the financial support received from Universiti Kebangsaan Malaysia under the research grant UKM-DLP-2011-038.

\section{REFERENCES}

[1]. D. Molodtsov, Soft set theory-first results, Computers and Mathematics with Applications, vol. 37, no. 4-5, pp. $19-31,1999$.

[2] D. Chen, E. C. C. Tsang, D. S. Yeung and X. Wang, The parameterization reduction of soft sets and its application, Computers and Mathematics with Applications, 49: 757-763 2005.

[3]. P. K. Maji, A. R. Roy and R. Biswas, Soft set theory, Computers and Mathematics with Applications, 45(45): $555-562,2003$.

[4]. P. K. Maji, A. R. Roy and R. Biswas, An application of soft Sets in a decision making problem, Computers and Mathematics with Applications, 44(8-9): 1077 1083, 2002.

[5]. P. K. Maji, A. R. Roy and R. Biswas, Fuzzy soft sets, Journal of Fuzzy Mathematics, 9(3): 589 - 602, 2001.

[6]. R. Roy and P. K. Maji, A fuzzy soft set theoretic approach to decision making problems, Journal of Computational and Applied Mathematics, 203(2): $412-418,2007$

[7] Cagman, N., Citak, F. \& Enginoglu, S. Fuzzy parameterized fuzzy soft set theory and its applications. Turkish Journal of Fuzzy Systems, 1: 21-35, 2010.

[8] S. Alkhazaleh, A. R. Salleh and N. Hassan, Possibility Fuzzy Soft Set, Advances in Decision Sciences, 2011(2011) 18 pages.

[9] S. Alkhazaleh, A. R. Salleh and N. Hassan, Fuzzy parameterized interval- valued fuzzy soft set, Applied Mathematical Sciences, 5(67): 3335-3346, 2011.

[10] S. Alkhazaleh and A. R. Salleh, Soft expert sets, Advances in Decision Sciences, 2011(2011) 15 pages.

[11] A. R. Salleh, S. Alkhazaleh, N. Hassan and A. G. Ahmad, multiparameterized soft set, Journal of Mathematics and Statistics, 8(1): 92-97, 2012.

[12] S. Alkhazaleh , 2012. Soft sets and fuzzy soft sets: Some generalizations. $\mathrm{PhD}$ thesis, Universiti Kebangsaan Malaysia. 